\title{
Uso de insectos como alternativa en la nutrición avícola: revisión
}

\author{
Uso de insetos como alternativa na nutrição de aves: revisão \\ Use of insects as an alternative in poultry nutrition: review
}

Recibido: 24/02/2021 | Revisado: 03/03/2021 | Acepto: 08/03/2021 | Publicado: 14/03/2021

Fanny Leonila Velasquez Moreno

ORCID: https://orcid.org/0000-0001-6791-8455

Universidade Federal de Mato Grosso, Brasil

E-mail: morenovelasquezfanny @gmail.com

Ana Paula Silva Ton

ORCID: https://orcid.org/0000-0002-5826-1874

Universidade Federal de Mato Grosso, Brasil E-mail: anatonn4@hotmail.com

Cecilia María Guerra Rosa

ORCID: https://orcid.org/0000-0001-6842-3200 Centro Agronômico Tropical de Investigación y Enseñanza, Costa Rica E-mail: cecilia.guerra@catie.arc.cr

Leonardo Willian de Freitas

ORCID: https://orcid.org/0000-0002-5796-0403 Universidade Federal de Mato Grosso, Brasil E-mail: lwillianf86@gmail.com

\begin{abstract}
Resumen
La producción de insectos como fuente de nutrientes representa una alternativa de producción sostenible, atrayente y adecuada para satisfacer la creciente demanda de alimentos, especialmente para la nutrición animal. Estrategia nutricional que puede ser considerada principalmente por la industria alimentaria, ya que en comparación con otros alimentos de uso común como las harinas de origen animal y la harina de soja, puede aportar cantidades significativas de proteínas, lípidos, minerales, entre otros nutrientes. Estos insectos pueden ser alimentados con residuos agrícolas y desechos orgánicos, siendo esta una de las principales ventajas que refuerzan el uso de este ingrediente en la alimentación animal. Además, disminuyen costos de producción e intervienen en el desenvolvimiento de la microbiota benéfica, reducen la competencia por recursos alimenticios y actúan como inmunomoduladores. Actualmente, algunos estudios resaltan el uso de diversas harinas de insectos con la inclusión o sustitución total de las fuentes convencionales de proteína. Un ingrediente que ofrece resultados significativos de interés zootécnico, generando un impacto positivo en el sistema productivo, por lo cual merece destacarse. El objetivo de esta revisión de literatura es resaltar el uso de insectos en la nutrición animal, principalmente en la avicultura, como una alternativa económicamente viable para el productor en términos de reducción de costos sin afectar el desempeño productivo de los animales. En este sentido, destacar esta temática como una nueva perspectiva en la industria de la alimentación animal y el sector pecuario.
\end{abstract}

Palabras clave: Desempeño productivo; Inmunomodulador; Nutrición animal; Nutriente.

\section{Resumo}

A produção de insetos como fonte de nutrientes representa uma alternativa de produção sustentável, atrativa e adequada para atender à crescente demanda por alimentos, principalmente para nutrição animal. Estratégia nutricional que pode ser considerada principalmente pela indústria alimentícia, pois comparada a outros alimentos comumente utilizados como farinha animal e farelo de soja, pode fornecer quantidades significativas de proteínas, lipídios, minerais, entre outros nutrientes. Esses insetos podem ser alimentados com resíduos agrícolas e resíduos orgânicos, sendo esta uma das principais vantagens que reforçam a utilização deste ingrediente na alimentação animal. Além disso, reduzem os custos de produção e interferem no desenvolvimento da microbiota benéfica, reduzem a competição por recursos alimentares e atuam como imunomoduladores. Atualmente, alguns estudos destacam o uso de várias farinhas de insetos com a inclusão ou substituição total de fontes convencionais de proteína. Um ingrediente que oferece resultados significativos de interesse zootécnico, gerando impacto positivo no sistema de produção, pelo qual merece destaque. O objetivo desta revisão de literatura é destacar a utilização de insetos na nutrição animal, principalmente na avicultura, como uma alternativa economicamente viável para o produtor em termos de redução de custos sem afetar o desempenho produtivo dos animais. Nesse sentido, destaca-se esta questão como uma nova perspectiva na indústria de ração animal e no setor pecuário.

Palavras-chave: Desempenho produtivo; Imunomodulador; Nutrição animal; Nutrientes. 


\begin{abstract}
The production of insects as a source of nutrients represents a sustainable, attractive, and adequate production alternative to satisfy the growing demand for food, especially for animal nutrition. This is a nutritional strategy that can be considered mainly by the food industry since, compared to other commonly used foods such as animal flour and soybean meal, it can provide significant amounts of proteins, lipids, minerals, among other nutrients. These insects can be fed with agricultural residues and organic waste; being this one of the most important advantages that reinforces the use of this ingredient to feed animals. This strategy also reduces production costs and intervenes in the development of beneficial microbiota. Moreover, it reduces competition for food resources and insects act as immunomodulators. Currently, some studies highlight the use of various insect meals with the total inclusion or substitution of conventional sources of protein; an ingredient that offers significant results of zootechnical interest, generating a positive impact on the productive system, for which it deserves to be highlighted. The objective of this literature review is to highlight the use of insects in animal nutrition; mainly in aviculture as an economically viable alternative for the producer in terms of cost reduction, without affecting the productive performance of animals. In this sense, highlighting this issue as a new perspective in the animal feed industry and the livestock sector is of big importance.
\end{abstract}

Keywords: Animal nutrition; Immunomodulator; Nutrient; Productive performance.

\title{
1. Introducción
}

La población mundial está en constante crecimiento y el suministro de alimentos no es adecuado, de acuerdo con la Organización de las Naciones Unidas para la Agricultura y la Alimentación (FAO) y la Organización Mundial de la Salud (OMS) (2017), las proyecciones para las próximas décadas indican que el consumo diario per cápita de proteína animal ha aumentado en un $22 \%$ para el año 2030 y en un $25 \%$ para 2050 , incluso con un crecimiento económico reducido en todos los países en desarrollo.

Las opciones para obtener esta proteína animal serán más limitadas, debido al alto precio que registrará la carne como una de las principales fuentes de proteína. Las poblaciones más afectadas serían las de los llamados países del tercer mundo. Por lo tanto, es fundamental que el productor sea innovador, estratégico y alternativo en la búsqueda de fuentes de nutrientes más baratas para el desarrollo y rápido crecimiento de los animales de fundamental importancia en la nutrición humana (Van Huis et al., 2014).

En la actualidad, esos ingredientes o fuentes de nutrientes pueden ser adquiridos de una abundante especie de material vegetal, el aprovechamiento de residuos de industrias, súmese a esto la importancia que los insectos representan no solo en la nutrición humana, de acuerdo con relevantes estudios demuestran el impacto significativo como ingrediente en la nutrición animal.

Los insectos deben ser considerados una prioridad en la búsqueda de alimentos alternativos en la nutrición animal, ya que la composición química y el valor nutricional de algunas especies de insectos han sido investigadas en varios países del mundo, demostrando que pueden aportar cantidades significativas de proteína, lípidos, minerales, entre otros nutrientes (Boland et al., 2013; Valipour et al., 2015).

Entre algunos insectos utilizados en investigaciones de nutrición animal, resaltan especies como: el grillo (Gryllus assimilis), la larva de tenebrio (Tenebrio molitor) y el super gusano de la harina (Zophobas morio). Insectos que pueden ser criados en condiciones controladas, buscando determinar el perfil nutricional y la calidad proteica de estos insectos en el crecimiento y desempeño productivo de las aves (Nowak et al., 2016).

Por lo tanto, investigadores en el área de la producción animal han logrado demostrar que la harina de (Gryllus assimilis) es una fuente viable y de alto valor nutricional, una excelente alternativa en la nutrición de aves, cerdos, peces y mascotas (Henry et al., 2015). El contenido de proteína cruda varía del 40 al $60 \%$ en la materia seca, este valor depende del estado morfológico del insecto, la tecnología de producción y la composición de los alimentos para la producción de las larvas. Además, presentan otros nutrientes valiosos como el contenido de grasa que puede variar del 10 al $40 \%$ presente en todas las etapas de la vida del insecto (Makkar et al., 2014). 
Nutriente que puede ser utilizado como una alternativa para reemplazar fuentes tradicionales de proteínas como la harina de soja y las harinas de origen animal, además del contenido de proteínas, alimentar pollos de engorde y otras aves de interés zootécnico con insectos presenta ciertos beneficios o ventajas, por ej. la presencia de quitina, un polisacárido (carbohidrato complejo) que se encuentra en el exoesqueleto de los insectos, puede ayudar a estimular el sistema inmunológico de las aves, reduciendo así la necesidad de antibióticos (Nowak et al., 2016).

Los primeros estudios en evaluar la inclusión de insectos en la alimentación animal fueron realizados con peces, sobre todo en regiones de África y Asia. Las oportunidades que los insectos pueden ofrecer en la avicultura aún no se han aprovechado en su totalidad, por lo cual, se convierte en una alternativa de nutrición inexplorada. Sin embargo, la producción de insectos como ingrediente alimenticio debe aumentar en gran escala, manteniendo su calidad y reduciendo los costos de producción, con la finalidad de brindar una alternativa competitiva contra fuentes de proteína usadas comúnmente. Es necesario considerar ciertos factores que pueden estar vinculados en la producción de estos insectos, como la productividad de biomasa de cada especie, composición bromatológica, viabilidad económica, hábitos alimenticios y componentes antinutricionales presentes (Schiavone et al., 2016).

En la actualidad, existen datos limitados sobre el uso de insectos como fuente de proteína y energía en la avicultura, considerando que existe una amplia variedad de insectos comestibles que ya fueron investigados en cuanto a su composición bromatológica. Algunos de los cuales ya están en el mercado, que pueden ser implementados y probados en la nutrición de pollos de engorde y otras especies de aves o animales que son de importancia para el consumo humano (Van Huis et al., 2013).

En este sentido, es necesario desarrollar prácticas más sostenibles por parte de la industria alimentaria. Como la entomofagia que es una práctica común en muchos países del mundo, existen más de 2000 especies de insectos que se pueden utilizar como alimento, cuyo efecto en la nutrición animal debe ser investigado y promovido constantemente, como nuevas alternativas para el desarrollo continuo de la avicultura (Jackowski et al., 2015).

\section{Metodología}

Se realizó una revisión narrativa (Vosgerau \& Romanowski, 2014), através de la investigación bibliográfica en diferentes bases de indexación de documentos, artículos y libros, sobre temas relacionados con el uso de insectos en la nutrición de las aves. Se utilizaron las palabras clave "insectos comestibles", "insectos en aves" e "insectos en nutrición animal" y las bases de indexación fueron: Scopus, Scielo, Google Scholar, Pub Med y Web of Science. No se definió un marco de tiempo, ya que buscaba mostrar la evolución del desarrollo de la investigación sobre el uso de insectos en la nutrición de las aves.

En la descripción narrativa se analizan producciones bibliográficas en un área determinada, aportando el estado del arte sobre un tema específico, evidenciando nuevas ideas, métodos os subtemas que han recibido más o menos énfasis en la literatura seleccionada (Vosgerau \& Romanowski, 2014).

\section{Desarrollo}

\subsection{Historia de la entomofagia}

Es evidente que tanto los ancestros del homo sapiens y las comunidades primitivas usaban insectos como fuente de alimento y nutrientes, antes de que los humanos adquirieran herramientas para cazar y recolectar alimentos, siendo China uno de los países pioneros en aprovechar esta fuente de nutrientes. Desde tiempos antiguos el uso de larvas y pupas de avispas en la cocina china fue registrado en el libro Tang Dynastyera del arte culinario chino. Los antiguos romanos también consumían insectos, ya que las larvas del gran escarabajo Capricornio (Cerambyx) eran especialmente populares en platillos servidos para 
personas del imperio. Por supuesto, en los libros sagrados de las religiones cristiana, judía e islámica, también se refieren a esta práctica conocida como entomofagia.

En este contexto, los insectos como alimento para humanos y animales emergen como un tema de especial relevancia en el siglo XXI, principalmente debido a los crecientes costos de las proteínas de origen animal, la inseguridad alimentaria, presiones ambientales y el crecimiento poblacional. Conjunto de factores por los cuales los insectos se presentan como una alternativa prometedora debido a su valor nutricional, beneficio para el medio ambiente y fácil producción (Banjo et al., 2006).

En el mundo existen alrededor de 1,5 millones de especies animales, de las cuales los insectos representan casi dos tercios de este total $(950$ mil). El crecimiento del sector de producción de proteína animal dependerá en gran medida de la existencia de ingredientes para la formulación de alimento animal. La oportunidad para que los insectos atiendan esta creciente demanda y reemplacen las fuentes tradicionales de proteínas y energía para la nutrición animal es muy acertada (Van Huis et al., 2013).

Entre la gran variedad de insectos comestibles que existen, su valor nutricional es muy variable, incluso dentro del propio grupo de especies, estado morfológico del insecto, su hábitat y alimentación. La digestibilidad y el valor nutricional de los insectos dependen de la especie a utilizar y pueden variar del 40 al 60\% de la proteína bruta en la materia seca (Schabel, 2010). Para referirse algunas especies como la mosca doméstica (Musca domestica), presentan valores de proteína bruta que oscilan entre 43 y 68\%, la chinche de la harina (Tenebrio molitor) que pertenece a la orden coleóptera presenta valores entre 44 y $69 \%$ muy cercanos a los encontrados en la harina de soja 45 a 68\% (Veldkamp et al., 2012).

\subsection{Ventajas del uso de insectos en la alimentación animal}

Según Veldkamp et al. (2012), algunas ventajas por las cuales el uso de insectos es una fuente de alimento alternativa y viable:

- Los insectos pueden ser producidos con subproductos o residuos orgánicos y biorresiduos, reduciendo así la contaminación ambiental;

- Emite significativamente menos gases de efecto invernadero;

- Necesitan menos recursos naturales como agua y suelo en comparación con otras especies agropecuarias;

- Una conversión alimenticia eficaz;

- Presentan un bajo riesgo de transmisión de infecciones por zoonosis;

- Alta tasa de crecimiento y reproducción;

- Menor costo de producción

\subsection{Uso de insectos en la alimentación animal}

La producción de alimentos de origen animal se ha tornado cada vez más costosa en términos económicos y medioambientales. Esta situación se debe principalmente al aumento de la demanda, debido al crecimiento poblacional, lo que genera un incremento en la demanda e ingesta de productos derivados de animales.

Se cree que la demanda de productos cárnicos aumentará considerablemente en un 58\% entre 2020 y 2050 . Por lo tanto, la producción mundial de concentrados para animales aumentara alrededor de mil millones de toneladas por año, incluidos 600 millones de toneladas de concentrado compuesto (FAO, 2004).

La demanda por ingredientes destinados para nutrición animal ha aumentado y se espera que la población humana, los recursos naturales y el ecosistema generen una presión significativa para producir proteína animal. Son necesarios cambios e innovaciones en los sistemas de producción avícola, porcino y ganaderos con el objetivo de satisfacer las demandas actuales y 
futuras de productos de origen animal. En este contexto, la investigación y la implementación comercial de nuevos alimentos (especialmente los ricos en proteínas) son necesarios para una nutrición animal más sostenible (Veldkamp et al., 2012).

Dado que en la mayoría de los sistemas de producción pecuaria la alimentación representa entre el 50 y el 70\% de los costos, en base a esto se buscan alternativas para mantener y mejorar el desempeño productivo de los animales que permitan al productor reducir en esta inversión. En este sentido, los insectos son una excelente alternativa en la alimentación de algunas especies de interés zootécnico como: aves, peces, cerdos, entre otros. Se considera que los insectos son ricos en proteínas, grasas y contenido mineral, el cual varía en función de la especie y la etapa del ciclo de vida en la que son utilizados, por lo tanto, puede mejorar el rendimiento y los índices zootécnicos de animales destinados a la producción, además de ser una alternativa menos demandante en cuanto a recursos naturales y menos generadores de residuos y gases de efecto invernadero (Verbeke et al., 2015).

Muchas especies de peces se alimentan de insectos, por lo que esta alternativa de alimentación ya está siendo explorada en la acuicultura. Por ejemplo, las larvas de la mosca soldado negra ya se utilizan con éxito para alimentar al dorado (Pargus major), la trucha arco iris (Oncorhynchus mykiss), el bagre de canal (Ictalurus punctatus) y la tilapia azul (Oreochromis aureus). En la nutrición de la trucha arco iris, las larvas pueden substituir hasta 25\% de harina de pescado y $38 \%$ el aceite de pescado (Ido et al., 2015).

La harina de la mosca soldado negra ha demostrado ser un ingrediente adecuado en las dietas de los cerdos en crecimiento debido a su perfil de aminoácidos, lípidos y calcio. Existen pocos y antiguos estudios sobre el uso de insectos en la alimentación avícola y porcina, sin embargo, esos estudios han mostrado excelentes resultados en relación a la tasa de crecimiento y rendimiento productivo (Opoku et al., 2018).

También cabe señalar que un número considerable de animales de diferentes especies, tanto en zoológicos como animales de compañía, se alimentan de insectos. Entre los insectos más utilizados como ingredientes alternativos en la nutrición animal y humana a nivel mundial se encuentran: escarabajos (Coleoptera 31\%), orugas (Lepidoptera 18\%), abejas, avispas y hormigas (Hymenoptera 14\%), saltamontes, grillos (Orthoptera 13\%), cigarras, chicharras, gusanos (Hemiptera 10\%), termitas (Isoptera 3\%), libélulas (Odonata 3\%), moscas (Diptera 2\%) y otras especies (5\%) (Reis \& Dias, 2020).

\subsection{Uso de insectos en la nutrición de aves}

Los requerimientos nutricionales de animales monogástricos incluyen mayor calidad y cantidad de proteína en la dieta desde el punto de vista nutricional, por lo tanto, las fuentes proteicas deben tener un alto contenido de este nutriente, un adecuado perfil de aminoácidos, alta digestibilidad, buena palatabilidad y sin factores antinutricionales (Animal Feed Resource Information System [AFRIS], 2015).

Actualmente, las principales fuentes de proteína para la alimentación y nutrición de aves son la harina de pescado y la harina de soja, productos vinculados a problemas ambientales en relación con el uso de agroquímicos para su producción que promueven la degradación ambiental, así como, el aumento de los precios de estos productos debido a la alta demanda y la inestabilidad productiva cada año por diversos factores.

La búsqueda de proteínas alternativas y sostenibles es un tema de gran importancia y necesita soluciones a corto plazo, lo que convierte a los insectos en una opción de alimentación cada vez más atractiva para las aves. Dado que en el comportamiento natural de las aves silvestres y domésticas está presente el consumo de insectos, especialmente en las primeras etapas de la vida (Makinde, 2015).

La alta exigencia nutricional de pollos de engorde, gallinas de postura y demás especies de aves y el bajo margen de ganancias que obtiene el productor, son factores que promueven la búsqueda por ingredientes de alto valor nutricional. Como una de esas alternativas, algunos estudios en el área avícola demuestran que las larvas de la mosca doméstica común (Musca 
doméstica) son una alternativa muy utilizada como alimento para pollos de engorde. Una opción alimentaria que tuvo un impacto positivo en países como: Nigeria, Rusia, Corea del Sur, India, China, Camerún entre otros (Hwangbo et al., 2009).

Las larvas de la mosca doméstica se utilizan para alimentar a pollos de engorde, tanto en forma fresca como seca, se considera que en estado seco existen algunas ventajas, ej. facilita el transporte, aumenta el tiempo de almacenamiento de este ingrediente y puede reemplazar a otros ingredientes tradicionales en las dietas, como harina de pescado, harina de soja, harina de carne y huesos entre otros, permitiendo mantener un buen desempeño productivo en pollos de engorde. En Corea del Sur, las larvas de insectos son de gran importancia, se ha demostrado que los pollos alimentados con este ingrediente mejoran las características de calidad de la carne y aumenta el peso vivo en 10 a 15\% (Hwangbo et al., 2009).

En India, la alimentación convencional representa aproximadamente el $60 \%$ del costo total de la cría de aves, pero la escasez de fuentes de alimentos como el maíz y la soja dificulta la producción de alimento y esta tendencia o comportamiento solo incrementa (Medhi, 2011). Por lo tanto, la recolección de algunos insectos que actúan como plagas agrícolas se convierte en una fuente utilizada como ingredientes en la nutrición animal, reduciendo así la incorporación de pesticidas nocivos por parte del ser humano, generando un impacto positivo al ambiente y utilizando esta fuente de nutrientes como una alternativa de alimentación animal.

Al utilizar algunos insectos como alternativa en la nutrición animal, varios investigadores se han centrado en estudiar la composición nutricional de la mayoría de las especies de esos insectos utilizados en las dietas, incluidas cuatro especies de acrididas: langostas comunes (Oxya fuscovittata), langostas comunes de la India (Acrida exaltata), langostas del arroz (Hieroglyphus banian) y saltamontes de cuernos cortos (Spathosternum prasiniferum prasiniferum) (Anand et al., 2008; AlQazzaz \& Ismail, 2015).

La mayoría de los estudios demuestran que los insectos son una alternativa viable que se caracteriza por tener un mayor contenido de proteína y grasa en comparación a fuentes tradicionales como la harina de soja y pescado, por lo cual, es una opción que puede ser implementada en la producción de alimentos para la nutrición animal (Anand et al., 2008).

Los insectos generalmente se utilizan poco en la producción comercial de alimentos, pero algunos estudios estiman que la calidad de la proteína del insecto tiene potencial como ingrediente alimentario. Muchas aves de compañía se consideran fitófagas y entomófagas. En la práctica, generalmente no hay aves que se alimenten solo de materiales vegetales, debido a que la mayoría de esas aves necesitan proteínas, por lo que se las consideró entomófagas y por lo tanto el consumo y producción de insectos es de mucho beneficio (Vidotto-Magnoni \& Carvalho, 2009).

Para mostrar la importancia que han logrado los insectos en la nutrición de las aves, se presentan algunos estudios y sus resultados: Aigbodion et al. (2012), realizaron un experimento con pollos de engorde donde se utilizaron cucarachas americanas (Periplaneta americana $L$.) en las dietas, con el objetivo de evaluar el crecimiento de los pollos a las ocho semanas de edad. Por lo tanto, los investigadores observaron que pollos alimentados con las dietas que incluían la harina del insecto presentaron los mejores resultados en cuanto a desempeño en comparación al tratamiento control que fue básicamente una dieta con ingredientes convencionales.

Estudios de López-Vergé et al. (2013), con el fin de verificar los efectos de la inclusión de larvas del gorgojo del maíz (Sitophilus zeamais Motschulsky) en la dieta de pollos de engorde. Como resultado, observaron que los pollos alimentados con la dieta que contenía larvas del insecto presentaron un mayor peso corporal final, mayor consumo y mejor conversión alimenticia en comparación al grupo de pollos que recibieron una dieta a base de harina de soja o dieta control, los resultados observados fueron justificados debido a una mayor proporción de proteína presente en las larvas.

Al trabajar con una dieta basada en harina de maíz y soja como alimento básico (control), y dietas suplementadas con 5, 10, 15 y 20\% de harina de la mosca soldado (Hermetia illucens) Dabbou et al. (2018), observaron que las dietas que contenían entre un 10 y un $15 \%$ de harina del insecto mejoraron la calidad de la canal, peso vivo y mayor consumo de 
concentrado, esos resultados fueron justificados por los autores debido a la excelente palatabilidad del alimento, en cuanto a los parámetros sanguíneos no presentaron diferencia significativa, por lo tanto, pollos que recibieron la dieta con la inclusión de $15 \%$ de la harina del insecto presentaron menor altura de las vellosidades intestinales, mayor profundidad de la cripta y menor relación altura-profundidad de vellosidades en comparación a los otros grupos.

Altmann et al. (2018), observaron que la inclusión de harinas de insectos en el concentrado puede influenciar la calidad de la carne de pollo, los que pollos que reciben dietas con harina de insecto presentan niveles de pH más estables en el filete de pechuga fresco hasta siete días después del empacado, en comparación con el filete de pechuga del grupo de pollos que reciben una dieta control (sin harina).

Estudio realizado por Benzertiha et al. (2020), para investigar el efecto de la suplementación de insectos ricos en grasas, como (Tenebrio molitor y Zophobas morio), en dietas balanceadas para evaluar el rendimiento, crecimiento y sistema inmunológico de pollos de engorde. En este estudio se evaluaron seis tratamientos, una dieta de control negativo, una dieta de control positivo con adición de antibiótico y cuatro dietas que contenían 0,2 y $0,3 \%$ de T. molitor y Z. morio. Observaron que la suplementación de insectos en pequeñas cantidades mejoraba la ganancia de peso diario, consumo de alimento y alteraba ciertas características del sistema inmunológico, como la producción de inmunoglobulinas e interleucinas en pollos de engorde.

En cuanto a aves de postura hay poca información, pero un estudio desarrollado por Yingchang et al. (1996), mostró que la sustitución del 5\% de la harina de pescado por harina de larva (T. molitor) resultó en un aumento del 2,4\% en la tasa de postura de las aves, justificaron que ese resultado es debido a una mayor cantidad de proteína y algunos minerales como cálcio procedentes de la harina del insecto.

Mwaniki et al. (2018), evaluaron la inclusión 7.5\% de harina de la mosca soldado negra (Hermetia illucens) en el concentrado de aves de postura, observaron que la producción y peso medio de los huevos fue semejante a los resultados observados con la dieta control ( $\sin$ harina de insecto). Pero la calidad de la cascara del huevo medida a través de la resistencia a quebrarse y la espesura aumentaron considerablemente con la inclusión de la harina, los autores justificaron los resultados debido a una mayor absorción de calcio en el intestino de las gallinas.

Otro estudio desarrollado por Zotte et al. (2019), evaluaron la inclusión de harina de la mosca soldado (Hermetia illucens) en codornices de postura, por lo cual no observaron diferencia significa en el desempeño de las aves, entre tanto las aves que recibieron dietas con harina del insecto presentaron mayor porcentaje de cascara, mayor pigmentación de la yema y mayor estabilidad oxidativa del producto, logrando un mayor tiempo de almacenamiento.

Hay pocos estudios sobre el tema en el área de la avicultura, sin embargo, estudios reflejan que pollos alimentados con harina de langostas sabrían mejor que los alimentados con concentrado comercial (Khusro et al., 2012). Además, se observó que la carne tiene menor contenido colesterol libre, mayor concentración de lípidos y fosfolípidos, aumento del potencial antioxidante y una vida útil del producto más prolongada (Sun et al., 2012).

\subsection{Procesamiento de insectos y diferentes tipos de productos.}

Una vez recolectados, los insectos mueren a través del proceso de liofilización, secado, ebullición o congelación. Se pueden procesar y consumir de tres formas:

1. Insectos enteros

2. En forma de harina

3. Extracto de proteína, grasa y quitina

Insectos enteros: En sistemas de producción orgánica que buscan alimentos de forma más natural, las larvas pueden ser proporcionadas vivas para los animales (Van Broekhoven et al., 2015). 
Harinas: Moler o triturar, manteniendo bajos niveles de humedad, es un método común para procesar una amplia variedad de alimentos. En forma de harina, los insectos se pueden mezclar mejor en la fabricación de concentrado logrando una mezcla más homogénea (Van Huis et al., 2015).

Extracto proteico: La separación de grupos de proteínas extraídas en función de su solubilidad en disolventes produce fracciones solubles e insolubles en agua, que pueden ser utilizadas para la incorporación específicas en las industrias de alimentos (Gasco et al., 2014).

Quitina: La quitina es el segundo polisacárido más abundante en la naturaleza, contiene nitrógeno y se encuentra comúnmente en organismos inferiores, como hongos, crustáceos e insectos. Tiene un papel importante en el sistema inmunológico aumentando la actividad de ciertas inmunoglobulinas e interleucinas (Veldkamp et al, 2012).

\section{Consideraciones Finales}

El uso de insectos en la alimentación animal como ingrediente proteico es un tema poco investigado y conocido en la industria alimentaria. Debido a las pocas investigaciones realizadas sobre el asunto en países subdesarrollados, presentan a los insectos como una nueva alternativa sustentable en la nutrición animal y amigable con los recursos naturales. Son investigaciones que han llamado la atención de la comunidad internacional para invertir cada vez más sobre la temática, y a través de estos trabajos se ha logrado demostrar el potencial nutricional de los insectos.

Por lo tanto, estos estudios, con el desarrollo de sistemas para la cría de insectos en gran escala, la actual crisis económica y el alza de los precios de los alimentos, ofrecen una perspectiva interesante sobre el uso de insectos para diferentes fines. Especialmente, en la nutrición animal, agricultura, obtención de aceites esenciales, producción de biodiesel y contribuyendo al equilibrio del medio ambiente. En base a esto, se comprueba que en los próximos años habrá un aumento significativo de la producción científica relacionada al uso de insectos en la alimentación animal como fuente competitiva y superior frente a las fuentes tradicionales de proteína.

Países de América central y Sudamérica tienen grandes expectativas para la producción de harina de insectos debido al clima y las variedades de insectos que su fauna posee, por ejemplo, Brasil país destacado como el $5^{\text {to }}$ mayor productor de seda del mundo, por lo que ya cuenta con residuos de larvas que pueden ser procesados o destinados directamente en la alimentación animal.

\section{Referencias}

Aigbodion, F. I., Egbon, I. N., \& Erukakpomren, E. A. (2012). Preliminary study on the entomophagous response of Gallus gallus domesticus (Galliformes: Phasianidae) to adult Periplaneta americana, International Journal of Tropical Insect Science, 32(3), 123-125. 10.1017/S1742758412000276

Al-Qazzaz, M., \& Ismail, D. B. (2015). Insect meal as a source of protein in animal diet. Animal Nutrition and Feed Technology, 16(3), 527-547. 10.5958/0974-181X.2016.00038.X

Altmann, B., Neumann, C., Velten, S., Liebert, F., \& Mörlein, D. (2018). Meat quality derived from high inclusion of a micro-alga or insect meal as an alternative protein source in poultry diets: a pilot study. Foods, 7(3), 34. 10.3390/foods7030034

Anand, H., Ganguly, A., \& Haldar, P. (2008). Potential value of acridids as high protein supplement for poultry feed. international Journal of poultry science, 7(7), 722-725. 10.3923/ijps.2008.722.725

Animal Feed Resource Information System. (2015). Food and Agriculture Organization of the United Nations, Rome, Italy.

Banjo, A. D., Lawal, O. A., \& Songonuga, E. A. (2006). The nutritional value of fourteen species of edible insects in South-western Nigeria. African Journal of Biotechnology, 5(3), 298-30. https://academicjournals.org/journal/AJB

Benzertiha, A., Kieronczyk, B., Kołodziejski, P., Pruszynska-Oszmałek, E., Rawski, M., Józefiak, D., \& Józefiak, A. (2020). Tenebrio molitor and Zophobas morio full-fat meals as functional feed additives affect broiler chickens' growth performance and immune system traits. Poultry Science, 99(1), 196-206. $10.3382 / \mathrm{ps} / \mathrm{pez} 450$

Boland, M. J., Rae, A. N., Vereijken, J. M., Meuwissen, M. P. M., Fischer, A. R. H., ..., Hendriks, W. H. 2013, The future supply of animal-derived protein for human consumption. Trends in Food Science \& Technology. 29(1), 62-73. 10.1016/j.tifs.2012.07.002 
Dabbou, S., Gai, F., Biasato, I., Capucchio, M. T., Biasibetti, E., Dezzutto, D., \& Schiavone, A. (2018). Black soldier fly defatted meal as a dietary protein source for broiler chickens: effects on growth performance, blood traits, gut morphology and histological features. Journal of Animal Science and Biotechnology, 9, 49. 10.1186/s40104-018-0266-9

Food and Agriculture Organization (2004). Protein Sources for the Animal Feed Industry, FAO Animal Production and Health Proceedings. Bangkok: FAO.

Food and Agriculture Organization/World Health Organization (2017). The State of Food and Agriculture: Leveraging Food Systems for Inclusive Rural Transformation. Rome, Italy: FAO.

Gasco, L., Belforti, M., Rotolo, L., Lussiana, C., Parisi, G., Terova, G., \& Gai, F. (2014). Mealworm (Tenebrio molitor) as a potential ingredient in practical diets for rainbow trout (Oncorhynchus mykiss). (p. 69-69) 1 st International conference "Insects to Feed the World", Netherlands. https://www.researchgate.net/

Henry, M., Gasco, L., Piccolo, G., \& Fountoulaki, E. (2015). Review on the use of insects in the diet of farmed fish: past and future. Animal Feed Science and Technology, 203, 1-22. 10.1016/j.anifeedsci.2015.03.001

Hwangbo, J., Hong, E. C., Jang, A., Kang, H. K., Oh, J. S., Kim, B. W., \& Park, B. S. (2009) Utilization of house fly-maggots, a feed supplement in the production of broiler chickens. Journal of Environmental Biology, 30(4), 609-614. http://jeb.co.in/

Ido, A., Iwai, T., Ito, K., Ohta, T., Mizushige, T., Kishida, T., ..., Miura, T. (2015). Dietary effects of housefly (Musca domestica). (Diptera: Muscidae) pupae on the growth performance and there sresistance against bacterial pathogen in red sea bream (Pagrus major) (Perciformes: Sparidae). Applied Entomology and Zoology, 50(2), 213-221. 10.1007/s13355-015-0325-z

Jackowski, J., Hurej, M., Rój, E., Popłoński, J., Kośny, L., \& Huszcza, E. (2015). Antifeedant activity of xanthohumol and supercritical carbon dioxide extract of spent hops against stored product pests. Bulletin of Entomological Research, 105(4), 456-461. 10.1017/S0007485315000255

Khusro, M., Andrew, N., \& Nicholas, A. (2012). Insects as poultry feed: a scoping study for poultry production systems in Aus tralia. World's Poultry Science Journal, 68(3), 435-446. 10.1017/S0043933912000554

López-Vergé, S., Barroeta, A. C., Riudavets, J., \& Rodríguez-Jerez, J. J. (2013). Utilization of Sitophilus zeamais (Motschulsky) larvae as a dietary supplement for the production of broiler chickens. Proceedings of The Nutrition Society, 72(OCE5), E315. 10.1017/S0029665113003492

Makinde, O. J. (2015). Maggot meal: a sustainable protein source for livestock production, a review. Advances in Life Science and Technology, 31, 35-41. https://iiste.org/Journals/index.php/ALST

Makkar, H. P. S., Tran, G., Heuzé, V., \& Ankers, P. (2014). State-of-the-Art on Use of Insects as Animal Feed. Animal Feed Science and Technology 197, pp. 1-33. 10.1016/j.anifeedsci.2014.07.008

Medhi, D. (2011). Effects of enzyme supplemented diet on finishing crossbred pigs at different levels of silk worm pupae meal in diet. Indian Journal of Field Veterinarians, 7(1), 24-26. https://www.cabdirect.org/

Mwaniki, Z., Neijat, M., \& Kiarie, E. (2018). Egg production and quality responses of adding up to $7.5 \%$ defatted black soldier fly larvae meal in a cornsoybean meal diet fed to Shaver White Leghorns from wk 19 to 27 of age. Poultry Science, 97(8), 2829-2835. 10.3382/ps/pey 118

Nowak, V., Persijn, D., Rittenschober, D., \& Charrondiere, U. R. (2016). Review of food composition data for edible insects. Food Chemistry, $193,39-46$. 10.1016/j.foodchem.2014.10.114

Opoku, O., Atuahene, C. C., Adjei, M. B., Quaye, B., \& Adu, M. A. (2018). Black soldier fly (hermetia illucens) larvae meal (bsflm) as alternative dietary protein source for broiler chickens; effects on growth performance, carcass and haematological parameters. Ghanaian Journal of Animal Science, 9(1), 25-31. https://www.researchgate.net/

Reis, T. L., \& Dias, A. C. C. (2020). Farinha de insetos na alimentação de não ruminantes, uma alternativa alimentar. Veterinária e Zootecnia, 27, 1-17. 10.35172/rvz.2020.v27.428

Schabel, H. G. (2010). Forest insects as food: A global review. Forest insects as food: Humans bite back, pp. 37-64.

Schiavone, A, Cullere, M., Marco, M., Meneguz, M., Biasato, I., Bergagna, S., \& Gasco, L. (2016). Partial or total replacement of soybean oil by black soldier fly larvae (Hermetia illucens $L$.) fat in broiler diets: effect on growth performances, feed-choice, blood traits, carcass characteristics and meat quality. Italian Journal of Animal Science, 16(1), 93-100. 10.1080/1828051X.2016.1249968

Sun, T., Long, R., \& Liu, Z. (2013). The effect of a diet containing grasshoppers and access to free-range on carcase and meat physicochemical and sensory characteristics in broilers. British poultry science, 54(1), 130-137. 10.1080/00071668.2012.756575

Valipour, M., Ziatabar, A. M., \& Raeini-sarjaz, M. (2015). Agricultural water management in the world during last half century. Archives of Agronomy and Soil Science. 61(5), 657-678. 10.1080/03650340.2014.944903

Van Broekhoven, S., Oonincx, D. G. A. B., Van Huis, A., Van Loon, J. J. A. (2015). Growth performance and feed conversion efficiency of three edible mealworm species (Coleoptera: Tenebrionidae) on diets composed of organic by-products. Journal of insect physiology, 73, 1-10. 10.1016/j.jinsphys.2014.12.005

Van Huis, A., Van Itterbeeck, J., Klunder, H., Mertens, E., Halloran, A., Muir, G., \& Vantomme, P. (2013). Edible insects: Future prospects for food and feed security (pp. 171). Rome: Food and Agriculture Organization of the United Nations.

Van huis, A., Vangurp, H., Dicke, M., Takken-kaminker, F., \& Blumenfeld-schaap, D. (2014). The insect cook book: Food for a sustainable planet (pp. 196). Columbia University Press.

Veldkamp, T., Van Duinkerker, G., Van Huis, A., Iakemond, C. M. M., Ottevanger, E., Bosch, G., \& Van Boekel, M. A. J. S. (2012). Insects as a Sustainable Feed Ingredient in Pig and Poultry Diets - a feasibility Study (pp. 48). Wageningen UR Livestock Research. 
Research, Society and Development, v. 10, n. 3, e25810313274, 2021

(CC BY 4.0) | ISSN 2525-3409 | DOI: http://dx.doi.org/10.33448/rsd-v10i3.13274

Verbeke, W., Sans, P., \& Van Loo, E. J. (2015). Challenges and prospects for consumer acceptance of cultured meat. Journal of Integrative Agriculture, 14(2), 285-294. 10.1016/S2095-3119(14)60884-4

Vidotto, A. P., \& Carvalho, E. D. (2009). Aquatic insects as the main food resource of fish the community in a Neotropical reservoir. Neotropical Ichthyology, 7(4), 701-708. 10.1590/S1679-62252009000400020

Vosgerau, D.S.R. \& Romanowski, J.P. (2014) Estudos de revisão: implicações conceituais e metodológicas. Revista Diálogo Educacional. 14(41), 165-189. 10.7213/dialogo.educ.14.041.DS08

Yingchang, W., Yuntang, C., Xingrui, L., Junmung, X., Qinsheng, D., Chag-an, Z. (1995). Study on the rearing larvae of Tenebrio moliter Linne and the effects of its processing and utilizing. Acta Agriculturae Universitatis Henanensis, 30(3) 288-292. https://europepmc.org/

Zotte, A. D., Singh, Y., Michiels, J., \& Cullere, M. (2019). Black soldier fly (Hermetia Illucens) as dietary source for laying quails: live performance, and egg physico-chemical quality, sensory profile and storage stability. Animals, 9(3):115. 10.3390/ani9030115 